\title{
DESENVOLVIMENTO DAS VERSÕES PORTU- GUESAS DOS QUESTIONÁRIOS FRAIL SCALE E SARC-F: FERRAMENTAS DE RASTREIO PARA A FRAGILIDADE FÍSICA E SARCOPENIA
}

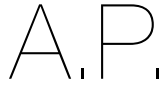

ARTIGO PROFSSIONAL

1 Faculdade de Ciências da Nutrição e Alimentação da Universidade do Porto, Rua do Campo Alegre, $n^{\circ} 823$,

4150-180 Porto, Portugal

2 Gripwise Tech, UPTEC Mar - sala F1,

Avenida da Liberdade,

4450-718 Leça da

Palmeira, Portugal

${ }^{3}$ Departamento de Biomedicina, Unidade

de Bioquímica da

Faculdade de Medicina

da Universidade do Porto,

Rua Dr. Plácido da Costa,

4200-450 Porto, Portugal

${ }^{4}$ I3S - Instituto de Investigação e Inovação em Saúde da Universidade do Porto,

Rua Alfredo Allen, n. ${ }^{\circ} 208$, 4200-135, Porto, Portuga

5 Faculdade de Ciências da Saúde, Universidade Fernando Pessoa,

Rua Carlos da Maia, n. ${ }^{\circ} 296$ 4200-150 Porto, Portugal

${ }^{6}$ Centro de Inovação em Tecnologias e Cuidados da Saúde (ciTechCare), Rua de Santo André 66-68, Campus 5 Politécnico de Leiria, 2410-541 Leiria, Portugal

*Endereço para correspondência:

Teresa F Amara

Faculdade de Ciências da Nutrição e Alimentação d Universidade do Porto, Rua do Campo Alegre, n. ${ }^{\circ} 823$, 4150-180 Porto, Portugal tamaral@fcna.up.pt

Histórico do artigo:

Recebido a 20 de março de 202 Aceite a 10 de agosto de 2021

\section{INTRODUÇÃO}

A população idosa, definida como indivíduos com idade igual ou superior a 65 anos (1), continua a aumentar globalmente (2). Como tal, deve ter-se especial atenção ao seu estado de saúde, introduzindo mecanismos e ferramentas que possibilitem o diagnóstico de morbilidades específicas deste grupo etário. Neste contexto, tem sido dada especial importância ao diagnóstico da fragilidade física e da sarcopenia, condições muito frequentes em pessoas idosas (3).

A fragilidade física trata-se de uma síndrome que se caracteriza por um declínio progressivo em múltiplos sistemas fisiológicos que resulta na diminuição das reservas de capacidade intrínseca, assim como no comprometimento da habilidade de manutenção da homeostasia, o que confere extrema vulnerabilidade a eventos causadores de stresse e aumenta o risco de consequências negativas para a saúde $(4,5)$. As complicações associadas à síndrome de fragilidade podem ser devastadoras para a população geriátrica, levando ao aumento de quedas, comprometimento do estado funcional e da mobilidade, decréscimo da função física e cognitiva, aumento do número de hospitalizações e, em casos mais graves, morte (6). Existe evidência que demonstra que o estado de fragilidade pode ser prevenido e reversível (7). Por conseguinte, a deteção precoce pode trazer benefícios ao paciente e permitir uma intervenção atempada $(6,8)$. De facto, é recomendado que se realize o rastreio da fragilidade física a todos os indivíduos com idade igual ou superior a 65 anos (7).

A FRAIL Scale foi desenvolvida pela International Association of Nutrition and Aging como uma ferramenta de rastreio de fragilidade de aplicação e interpretação simples $(9,10)$. Esta ferramenta é baseada tanto na abordagem do Fenótipo de Fragilidade proposto por Fried et al. (11), como na abordagem desenvolvida por Rockwood et al. 
que propõe o Frailty Index (12). Estas últimas ferramentas são mais difíceis de implementar em contexto clínico e comunitário, uma vez que têm um protocolo de aplicação mais complexo e requerem maior tempo de administração (8). Neste sentido, foi necessário criar uma ferramenta que permitisse identificar de forma rápida e simples os indivíduos em risco de fragilidade. A FRAIL Scale foi construída sob a forma de um questionário de auto reporte com resposta simples de "sim" ou "não". Inclui cinco componentes inseridos em três domínios: resistência e deambulação no domínio funcional, fadiga e perda de peso, inseridos no domínio biológico e, por último, em representação do domínio de acumulação de défices, o componente de doença (8, 10, 13). A possibilidade de existência da síndrome de fragilidade é indicada pela pontuação final, em que pontuações iguais e superiores a 3 indicam risco de fragilidade, pontuações de 1 ou 2 indicam risco de pré-fragilidade e 0 pontos indicam um estado de saúde robusto (9). A FRAIL Scale é considerada uma ferramenta válida na predição de incapacidade e mortalidade, sendo útil na identificação de potenciais áreas de tratamento (14). Quando comparada com outras estratégias, nomeadamente as supramencionadas, a FRAIL Scale é capaz de prever deficiências e mortalidade com precisão semelhante (15), apresentando alta especificidade e baixa a moderada sensibilidade (16).

No consenso do European Working Group on Sarcopenia in Older People 2 (EWGSOP 2), a sarcopenia é apresentada como uma doença muscular, definida pela perda de força muscular, associada a uma redução da qualidade e quantidade de massa muscular, sendo classificada como grave quando existe, concomitantemente, diminuição do desempenho físico (17). A sarcopenia está, do mesmo modo, relacionada com o declínio das atividades do dia-a-dia e da qualidade de vida, declínio da função cognitiva, depressão e aumento dos custos com a saúde (18). Tendo em conta a sua natureza reversível, é imperativo que se faça a sua deteção atempadamente de modo a estabelecer uma terapêutica adequada e identificar novas áreas de tratamento. Recomendações recentes apontam para a realização anual do rastreio desta doença em indivíduos com idade igual ou superior a 65 anos ou após algum evento clínico adverso major (19).

O diagnóstico da sarcopenia é complexo e difícil de implementar na prática clínica. Neste sentido, Malmstrom et al. desenvolveram o $S A R C-F(20)$, um método de rastreio simplificado para a sarcopenia em idosos, baseado nos critérios de EWGSOP. Segundo estes investigadores, o SARC-F deve ser incorporado no diagnóstico primário da sarcopenia, estando sugerido no algoritmo para deteção de casos, diagnóstico e determinação da gravidade da sarcopenia, criado pelos mesmos autores (17). O SARC- $F$ é um questionário de auto reporte, de fácil implementação em contexto clínico, concebido de modo a detetar alterações no estado de saúde associadas às consequências da sarcopenia (21). Permite determinar o nível de dificuldade percecionada pelo indivíduo para cinco componentes: força, assistência para caminhar, levantar da cadeira, subir escadas e quedas. A pontuação final varia entre 0 e 10, em que pontuações iguais ou superiores a 4, são sugestivas da presença de sarcopenia. Esta ferramenta já foi traduzida e validada para diversos idiomas, estando comprovada a sua alta especificidade e baixa a moderada sensibilidade (22). Diversos autores consideram este questionário como uma ferramenta válida e consistente para o rastreio da sarcopenia $(17,23)$, sendo reconhecida a sua grande utilidade como ferramenta de identificação para posterior confirmação do diagnóstico de sarcopenia (24).

Tendo em conta a grande aplicabilidade das ferramentas descritas, urge a necessidade da sua aplicação na população portuguesa. Para tal, é necessário que exista uma tradução dos questionários originais validada para a língua portuguesa. Neste sentido, são objetivos deste trabalho produzir uma tradução para a língua portuguesa das versões originais em inglês da FRAIL Scale e do SARC-F, bem como estudar a validade dessas traduções à luz das recomendações da PatientReported Outcome (PRO) Consortium (25). Pretende-se, também, verificar a validade linguística e cultural das traduções efetuadas, avaliar o grau de dificuldade de preenchimento e a compreensão, assim como a aplicabilidade destas duas ferramentas.

\section{METODOLOGIA}

A tradução da versão original e a validação da versão traduzida foi conduzida segundo as normas do PRO Consortium (25), que preconiza a realização dos seguintes passos: preparação, tradução, reconciliação, retrotradução, revisão da retrotradução, harmonização, revisão, entrevista cognitiva, revisão/análise, revisão final e documentação, relatório e arquivo/manutenção de registos.

Em seguida, encontra-se a descrição em detalhe da metodologia aplicada:

\section{Preparação}

O autor correspondente das ferramentas FRAIL Scale e SARC-F (Prof. John E. Morley), foi contactado e concedeu permissão para que fosse realizada a tradução e validação dos questionários para a língua portuguesa.

\section{Tradução}

Dois indivíduos nativos de língua portuguesa e fluentes em inglês, desenvolveram, em paralelo, duas traduções individuais das ferramentas FRAIL Scale e SARC-F, originalmente em língua inglesa, para português.

\section{Reconciliação}

Num painel constituído pelos investigadores e por uma nutricionista com vasta experiência na área, foram analisadas e discutidas as duas propostas. Para cada questão tentou-se uma maior aproximação à versão original. Não obstante, teve-se em consideração que a tradução efetuada não resultaria numa tradução literal, mas sim numa tradução conceitual adequada à população alvo das ferramentas. Desta forma, tendo em conta a equivalência semântica e sintática das propostas, criou-se uma versão primordial dos dois questionários onde foram integrados os pontos fortes de cada uma das traduções e sugestões consideradas pertinentes. De destacar que na ferramenta FRAIL Scale para a questão: "Qual é o seu peso com roupa, mas sem sapatos? Há um ano, em (mês, ano) quanto pesava (sem sapatos e com as roupas vestidas)?", considerando que na versão original da ferramenta o valor de 5 não estava contemplado em nenhum dos intervalos, o ponto de corte usado para a variação do peso foi alterado de $>5$ (versão original) para $\geq 5$ tendo em conta a escala de Fried et al. (11) para a fragilidade.

\section{Retrotradução}

A versão resultante foi enviada a um indivíduo nativo de língua inglesa, que não tinha qualquer conhecimento da versão original das ferramentas. Deste modo, procedeu-se à tradução da versão portuguesa criada para inglês, idioma original.

\section{Revisão da Retrotradução}

O painel comparou a tradução gerada com a versão original e verificou que existiam algumas diferenças entre ambas. A maioria das diferenças encontradas não foi considerada relevante, uma vez que não induziam discrepância ao nível de equivalência conceitual. No entanto, três questões foram verificadas tendo em conta que levantaram alguma 
ambiguidade perante a versão original. Na terceira questão do questionário $S A R C$ - $F$ procedeu-se à alteração da palavra "assistência" para a palavra "ajuda", atendendo a que este último termo corresponde a uma tradução mais correta. No questionário FRAIL Scale procedeu-se à alteração de duas questões. Na primeira questão, alterou-se a pergunta e as respetivas opções de resposta, dado que a versão portuguesa induzia uma resposta em termos de frequência e a versão original pretendia obter uma resposta referente à duração no tempo. Na última questão, verificou-se incongruência entre a tradução gerada ("What is your weight") e o original ("How much do you weight"), no entanto, por uma questão cultural e de equivalência conceitual, optouse por não realizar a alteração na versão portuguesa.

\section{Harmonização}

Após resolver as discrepâncias encontradas, o painel verificou a equivalência conceitual entre as versões originais e a versão portuguesa, fazendo as alterações necessárias. Alcançado o consenso, criou-se a versão pré-final de cada uma das duas ferramentas.

\section{Revisão}

O painel enviou a versão pré-final a duas nutricionistas experientes na área com nacionalidade portuguesa e fluentes em inglês, para que fosse feita uma revisão da tradução e verificada a coerência gramatical e conceitual. O painel de investigadores analisou as sugestões e fez as alterações que considerou mais pertinentes, tendo em conta o alcance de uma tradução fiel das ferramentas, adaptada ao contexto onde as mesmas serão aplicadas. No total, foram sugeridas seis alterações conceituais, das quais foram realizadas três. No questionário $S A R C-F$ foi feita uma alteração conceitual, visto que esta implicava uma maior coerência ao longo do questionário. No questionário FRAIL Scale, o termo "além de" foi substituído por "exceto" no seguimento da tradução do termo "other than". A alteração foi realizada de modo a não causar ambiguidade na interpretação da questão referida. O termo "cancro da pele minor" resultante da tradução original de "minor skin cancer" foi alterado para "cancro da pele não-melanoma". A alteração foi verificada por uma médica especialista em oncologia médica, que referiu ser este o termo mais correto e usado na prática clínica. As restantes três sugestões não foram efetuadas, uma vez que o painel concordou que, nestes casos, a versão original devia ser mantida de modo a obter uma maior aproximação ao questionário original, bem como maior congruência ao longo dos questionários. Além disso, caso algumas propostas fossem efetuadas, poderiam originar discrepância na interpretação das questões.

\section{Entrevista Cognitiva}

Iniciou-se um estudo piloto no qual se realizou a aplicação da versão pré-final dos questionários. O estudo teve como objetivos verificar o grau de dificuldade de preenchimento, a facilidade de compreensão dos questionários e a aplicabilidade das traduções geradas. Os questionários foram aplicados presencialmente ou por chamada telefónica pelos três investigadores do painel. A amostra de conveniência foi constituída por nove indivíduos, correspondentes à população alvo das ferramentas, com idades compreendidas entre os 61 e os 87 anos que não se encontravam institucionalizados. Cada indivíduo selecionado respondeu a cada um dos questionários. No total, obtiveram-se 18 questionários válidos, 9 correspondentes ao SARC F e 9 correspondentes ao FRAIL Scale, não havendo qualquer recusa na participação. Para a caracterização da amostra recolheu-se informação sobre sexo, idade, presença de patologias e o meio onde o inquirido se encontra. Dos 9 inquiridos, 7 eram do sexo feminino e 2 do sexo masculino. As características sociodemográficas da amostra selecionada encontram-se descritas na Tabela 1. A proporção de indivíduos com sinais sugestivos de sarcopenia pela aplicação do $S A R C-F$ foi de $44,4 \%$, ao passo que a proporção de indivíduos identificados em risco de fragilidade ou de pré-fragilidade pela aplicação da FRAIL Scale foi de 22,2\% para ambos os casos (Tabela 2).

\section{Revisão/Análise}

Terminada a ministração das ferramentas, foram recolhidas sugestões de melhoria, identificadas expressões e/ou palavras que pudessem gerar dúvidas aquando da futura aplicação das ferramentas, assim como analisados os seus pontos fortes e fracos. Os questionários tiveram boa aceitabilidade e aplicabilidade não tendo havido qualquer relutância à sua aplicação. As sugestões de melhoria foram analisadas pelo painel, que decidiu aplicar apenas uma alteração na classificação da "fadiga" na questão 1 da ferramenta FRAIL Scale de modo a tornar a resposta mais percetível, mantendo a escala de quantificação no tempo.

\section{Revisão Final e Documentação}

As ferramentas foram revistas de modo a verificar a existência de possíveis erros ortográficos, gramaticais e de sintaxe. O painel considerou como finalizado todo o processo de tradução apresentando as versões portuguesas da FRAIL Scale e do SARC-F no Anexo 1 e 2, respetivamente.

\section{Relatório}

Produção do relatório final enquadrado neste documento.

\section{Arquivo/Manutenção de Registos}

O painel guardou todas as versões realizadas ao longo do processo e registou todas as alterações e sugestões de melhoria. Do mesmo modo, encontram-se arquivados os resultados da aplicação das ferramentas.

\section{Tabela 1}

Características sociodemográficas da amostra de conveniência

\begin{tabular}{|c|c|}
\hline VARIÁVEL & MEDIANA (AIQ) OU n (\%) \\
\hline Idade (anos) & $78,0(12,0)$ \\
\hline Sexo Feminino & $7(77,8)$ \\
\hline \multicolumn{2}{|l|}{ Patologias } \\
\hline Hipertensão & $6(66,7)$ \\
\hline Artrite reumatoide & $2(22,2)$ \\
\hline Diabetes & $2(22,2)$ \\
\hline Osteoporose & $1(11,1)$ \\
\hline Doença pulmonar crónica & $1(11,1)$ \\
\hline Angina & $1(11,1)$ \\
\hline Doença renal & $1(11,1)$ \\
\hline Psoríase & $1(11,1)$ \\
\hline Bócio & $1(11,1)$ \\
\hline Demência & $1(11,1)$ \\
\hline Parkinson & $1(11,1)$ \\
\hline Insuficiência cardíaca & $1(11,1)$ \\
\hline Dislipidemia & $1(11,1)$ \\
\hline
\end{tabular}

\section{Tabela 2}

Características sociodemográficas da amostra de conveniência

\begin{tabular}{|c|c|c|c|c|}
\hline & & \multicolumn{2}{|c|}{ SARC-F $\mathrm{n}(\%)$} & \multirow[b]{2}{*}{ TOTAL } \\
\hline & & $\begin{array}{l}\text { SUGESTIVO DE } \\
\text { SARCOPENIA }\end{array}$ & $\begin{array}{l}\text { SEM SINAIS DE } \\
\text { SARCOPENIA }\end{array}$ & \\
\hline \multirow{4}{*}{$\begin{array}{l}\text { FRAIL Scale } \\
\mathrm{n}(\%)\end{array}$} & Fragilidade & $2(22,2)$ & $1(11,1)$ & $3(33,3)$ \\
\hline & Pré-fragilidade & $2(22,2)$ & $2(22,2)$ & $4(44,4)$ \\
\hline & Robusto & $0(0)$ & $2(22,2)$ & $2(22,2)$ \\
\hline & TOTAL & $4(44,4)$ & $5(55,6)$ & \\
\hline
\end{tabular}




\section{ANÁLISE CRÍTICA}

Através do presente trabalho, foram desenvolvidas versões portuguesas das ferramentas FRAIL Scale (6) e SARC-F (20). A utilização das normas do PRO Consortium (25) permitiu criar versões válidas e adaptadas para a língua portuguesa, mantendo a equivalência e conceitos das ferramentas originais.

De forma a estudar a validade das ferramentas, as mesmas foram aplicadas a uma amostra de conveniência, a qual não mostrou qualquer relutância à sua aplicação. As versões produzidas mostraram-se de fácil compreensão e aplicabilidade, rápida administração, demonstrando boa aceitabilidade e validade aparente.

Será importante referir que as ferramentas FRAIL Scale (6) e SARC-F (20) são baseadas no autorrelato de pessoas com idades iguais ou superiores a 65 anos, e que não está previsto o rastreio a alterações da função cognitiva, que poderiam comprometer a validade da avaliação. Também, nas publicações originais, não estão definidos os prérequisitos neste domínio, para a aplicação destas ferramentas. Uma explicação possível, será o facto de estas ferramentas serem aplicadas por profissionais de saúde, que conhecerão a capacidade cognitiva e mental das pessoas rastreadas.

As versões Portuguesas das ferramentas FRAIL Scale (6) e SARC-F (20) desenvolvidas podem ser relevantes no rastreio da fragilidade e da sarcopenia em ambiente hospitalar, nos cuidados de saúde primários e na comunidade, tanto em Portugal como noutros países de língua portuguesa. Tendo em consideração que a fragilidade física e a sarcopenia são indicativas de um grau acrescido de incapacidade e vulnerabilidade, espera-se que a utilização destas ferramentas permita a identificação precoce da população em risco e, consequentemente, o diagnóstico precoce destas condições e a intervenção atempada. Desta forma, poderão ser implementadas medidas com vista a impedir a evolução e a deterioração do estado nutricional e do quadro clínico destes indivíduos.

\section{AGRADECIMENTOS}

Ana Rita Sousa-Santos recebeu uma bolsa de doutoramento (referência: SFRH/BD/138362/2018) financiada pela Fundação para a Ciência e a Tecnologia (FCT), I.P., no âmbito do Programa Operacional Capital Humano $(\mathrm{POCH})$.

\section{REFERÊNCIAS BIBLIOGRÁFICAS}

1. Organisation for Economic Co-operation and Development (OECD). Elderly population (indicator). 2021. Disponível em: https://data.oecd.org/pop/elderly-population.htm. 2. Department of Economic and Social Affairs PD. World Population Ageing 2019: Highlights (ST/ESA/SER.A/430). United Nations 2019.

3. Tamura Y, Ishikawa J, Fujiwara Y, Tanaka M, Kanazawa N, Chiba Y, et al. Prevalence of frailty, cognitive impairment, and sarcopenia in outpatients with cardiometabolic disease in a frailty clinic. BMC Geriatr. 2018; 18(1):264.

4. Morley JE, Vellas B, Abellan van Kan G, Anker SD, Bauer JM, Bernabei R, et al. Frailty Consensus: A Call to Action. J Am Med Dir Assoc. 2013; 14(6):392-97.

5. World Health Organization (WHO). WHO clinical consortium on healthy ageing: topic focus: frailty and intrinsic capacity: report of consortium meeting, 1-2 December 2016 in Geneva, Switzerland. Geneva: World Health Organization; 2017. Disponível em: https://apps.who.int/iris/handle/10665/272437.

6. Clegg A, Young J, lliffe S, Rikkert MO, Rockwood K. Frailty in elderly people. The Lancet. 2013; 381(9868):752-62.

7. Dent E, Morley JE, Cruz-Jentoft AJ, Woodhouse L, Rodríguez-Mañas L, Fried LP, et al. Physical Frailty: ICFSR International Clinical Practice Guidelines for Identification and Management. J Nutr Health Aging. 2019; 23(9):771-87.

8. Aprahamian I, Cezar NOC, Izbicki R, Lin SM, Paulo DLV, Fattori A, et al. Screening for Frailty With the FRAIL Scale: A Comparison With the Phenotype Criteria. J Am Med
Dir Assoc. 2017; 18(7):592-96

9. Morley JE, Malmstrom TK, Miller DK. A simple frailty questionnaire (FRAIL) predicts outcomes in middle aged African Americans. J Nutr Health Aging. 2012; 16(7):601-8. 10. Abellan van Kan G, Rolland YM, Morley JE, Vellas B. Frailty: toward a clinical definition. J Am Med Dir Assoc. 2008; 9(2):71-2.

11. Fried LP, Tangen CM, Walston J, Newman AB, Hirsch C, Gottdiener J, et al. FraiIty in older adults: evidence for a phenotype. J Gerontol A Biol Sci Med Sci. 2001; 56(3):M146-56.

12. Rockwood K, Stadnyk K, MacKnight C, McDowell I, Hébert R, Hogan DB. A brief clinical instrument to classify frailty in elderly people. Lancet. 1999; 353(9148):205-6. 13. Abellan van Kan G, Rolland Y, Bergman H, Morley JE, Kritchevsky SB, Vellas B. The I.A.N.A Task Force on frailty assessment of older people in clinical practice. J Nutr Health Aging. 2008; 12(1):29-37.

14. Susanto M, Hubbard RE, Gardiner PA. Validity and Responsiveness of the FRAIL Scale in Middle-Aged Women. J Am Med Dir Assoc. 2018; 19(1):65-69.

15. Malmstrom TK, Miller DK, Morley JE. A comparison of four frailty models. J Am Geriatr Soc. 2014; 62(4):721-6.

16. Thompson MQ, Theou O, Tucker GR, Adams RJ, Visvanathan R. FRAIL scale: Predictive validity and diagnostic test accuracy. Australas J Ageing. 2020; 39(4):e529-e36. 17. Cruz-Jentoft AJ, Bahat G, Bauer J, Boirie Y, Bruyère O, Cederholm T, et al. Sarcopenia: revised European consensus on definition and diagnosis. Age Ageing. 2019; 48(1):16-31.

18. Ida S, Kaneko R, Murata K. SARC-F for Screening of Sarcopenia Among Older Adults: A Meta-analysis of Screening Test Accuracy. J Am Med Dir Assoc. 2018; 19(8):685-89.

19. Dent E, Morley JE, Cruz-Jentoft AJ, Arai H, Kritchevsky SB, Guralnik J, et al. International Clinical Practice Guidelines for Sarcopenia (ICFSR): Screening, Diagnosis and Management. J Nutr Health Aging. 2018; 22(10):1148-61.

20. Malmstrom TK, Morley JE. SARC-F: a simple questionnaire to rapidly diagnose sarcopenia. J Am Med Dir Assoc. 2013; 14(8):531-2.

21. Malmstrom TK, Miller DK, Simonsick EM, Ferrucci L, Morley JE. SARC-F: a symptom score to predict persons with sarcopenia at risk for poor functional outcomes. J Cachexia Sarcopenia Muscle. 2016; 7(1):28-36.

22. Bahat G, Yilmaz O, Kilıc C, Oren MM, Karan MA. Performance of SARC-F in Regard to Sarcopenia Definitions, Muscle Mass and Functional Measures. J Nutr Health Aging. 2018; 22(8):898-903.

23. Parra-Rodríguez L, Szlejf C, García-González Al, Malmstrom TK, Cruz-Arenas E, Rosas-Carrasco O. Cross-Cultural Adaptation and Validation of the Spanish-Language Version of the SARC-F to Assess Sarcopenia in Mexican Community-Dwelling Older Adults. J Am Med Dir Assoc. 2016; 17(12):1142-46.

24. Kera T, Kawai H, Hirano H, Kojima M, Watanabe Y, Motokawa K, et al. Limitations of SARC- $F$ in the diagnosis of sarcopenia in community-dwelling older adults. Arch Gerontol Geriatr. 2020; 87:103959.

25. Eremenco S, Pease S, Mann S, Berry P. Patient-Reported Outcome (PRO) Consortium translation process: consensus development of updated best practices. J Patient Rep Outcomes. 2017; 2(1):12. 


\section{ANEXO 1}

\section{FRAIL Scale}

\begin{tabular}{|c|c|c|}
\hline COMPONENTE & QUESTÃo & PONTUAÇÃOO \\
\hline Fadiga & $\begin{array}{l}\text { Quanto tempo se sentiu cansado nas últimas } 4 \\
\text { semanas? }\end{array}$ & $\begin{array}{l}1=\text { O tempo todo } \\
2=\text { A maior parte do tempo } \\
3=\text { Durante alguma parte do tempo } \\
4=\text { Pouco tempo } \\
5=\text { Nunca } \\
\text { Respostas de " } 1 \text { " ou " } 2 \text { " são pontuados como } 1 \text { e } \\
\text { todas as outras como } 0 .\end{array}$ \\
\hline Resistência & $\begin{array}{l}\text { Sozinho (a) e sem apoios, tem alguma dificuldade em } \\
\text { subir } 10 \text { degraus sem descansar? }\end{array}$ & $\begin{array}{l}1=\operatorname{Sim} \\
0=\text { Não }\end{array}$ \\
\hline Deambulação & $\begin{array}{l}\text { Sozinho (a) e sem apoios, tem alguma dificuldade em } \\
\text { caminhar várias centenas de metros? }\end{array}$ & $\begin{array}{l}1=\operatorname{Sim} \\
0=\text { Não }\end{array}$ \\
\hline Doença & $\begin{array}{l}\text { Para } 11 \text { doenças, os participantes são questionados: } \\
\text { "Algum médico alguma vez lhe disse que tem } \\
\text { [doença]?" } \\
\text { As doenças são: hipertensão, diabetes, cancro (exceto } \\
\text { cancro da pele não-melanoma), doença pulmonar } \\
\text { crónica, enfarte, insuficiência cardíaca congestiva, } \\
\text { angina, asma, artrite, AVC e doença renal. }\end{array}$ & $\begin{array}{l}1=\operatorname{Sim} \\
0=\text { Não } \\
\text { O número total de doenças }(0-11) \text { é recodificado como } \\
0-4=0 \text { e } 5-11=1\end{array}$ \\
\hline \multirow{2}{*}{ Perda de peso } & $\begin{array}{l}\text { Qual é o seu peso com roupa, mas sem sapatos? [peso } \\
\text { atual] }\end{array}$ & $\begin{array}{l}\text { Percentagem de variação de peso é calculada como: } \\
\text { [(peso há } 1 \text { ano - peso atual) / peso há } 1 \text { ano] }{ }^{*} 100 .\end{array}$ \\
\hline & $\begin{array}{l}\text { Há um ano, em (mês, ano), quanto pesava sem sapatos } \\
\text { e com as roupas vestidas? [peso há } 1 \text { ano] }\end{array}$ & $\begin{array}{l}\text { Perda de peso } \geq 5 \%=1 \\
\text { Perda de peso }<5 \%=0\end{array}$ \\
\hline
\end{tabular}

Traduzido e adaptado de: Morley JE, Malmstrom TK, Miller DK. A simple frailty questionnaire (FRAIL) predicts outcomes in middle aged African Americans. J Nutr Health Aging. 2012

\section{RESULTADO:}

Pontuação 0: Sem fragilidade

Pontuação 1 a 2: Risco de pré-fragilidade

Pontuação 3 a 5: Risco de fragilidade

\section{ANEXO 2}

\section{SARC-F}

\begin{tabular}{|c|c|c|}
\hline COMPONENTE & QUESTÃO & PONTUAÇÃO \\
\hline Força & $\begin{array}{l}\text { Qual é a dificuldade que tem em levantar e carregar } \\
4,5 \mathrm{~kg} ?\end{array}$ & $\begin{array}{l}\text { Nenhuma }=0 \\
\text { Alguma }=1 \\
\text { Muita ou incapaz }=2\end{array}$ \\
\hline Assistência para caminhar & $\begin{array}{l}\text { Qual é a dificuldade que tem em caminhar ao longo de } \\
\text { uma sala? }\end{array}$ & $\begin{array}{l}\text { Nenhuma }=0 \\
\text { Alguma }=1 \\
\text { Muita, usa apoios ou incapaz }=2\end{array}$ \\
\hline Levantar da cadeira & $\begin{array}{l}\text { Qual é a dificuldade que tem em transferir-se de uma } \\
\text { cadeira ou de uma cama? }\end{array}$ & $\begin{array}{l}\text { Nenhuma }=0 \\
\text { Alguma }=1 \\
\text { Muita ou incapaz sem ajuda }=2\end{array}$ \\
\hline Subir escadas & $\begin{array}{l}\text { Qual é a dificuldade que tem em subir um lanço de } 10 \\
\text { escadas? }\end{array}$ & $\begin{array}{l}\text { Nenhuma }=0 \\
\text { Alguma }=1 \\
\text { Muita ou incapaz }=2\end{array}$ \\
\hline Quedas & Quantas vezes caiu no último ano? & $\begin{array}{l}\text { Nenhuma }=0 \\
1-3 \text { quedas }=1 \\
4 \text { ou mais quedas }=2\end{array}$ \\
\hline
\end{tabular}

Traduzido e adaptado de: Malmstrom TK, Morley JE. SARC-F: a simple questionnaire to rapidly diagnose sarcopenia. J Am Med Dir Assoc. 2013.

\section{RESULTADO:}

Pontuação: 0 a 10 pontos.

Pontuações iguais ou superiores a 4 são sugestivas de sarcopenia e complicações adversas. 